\title{
Multicatheter interstitial brachytherapy versus stereotactic radiotherapy with CyberKnife for accelerated partial breast irradiation: a comparative treatment planning study with respect to dosimetry of organs at risk
}

\author{
András Herein ${ }^{1,2}$, Gábor Stelczer ${ }^{1,2}$, Csilla Pesznyák ${ }^{1,2}$, Georgina Fröhlich ${ }^{1,3}$, \\ Viktor Smanykó ${ }^{1}$, Norbert Mészáros ${ }^{1,4}$, Csaba Polgár ${ }^{1,4}$, Tibor Major ${ }^{1,4}$ \\ ${ }^{1}$ National Institute of Oncology, Radiotherapy Centre, Budapest, Hungary \\ ${ }^{2}$ Budapest University of Technology and Economic, Institute of Nuclear Techniques, Budapest, Hungary \\ ${ }^{3}$ Eötvös Loránd University, Faculty of Science, Budapest, Hungary \\ ${ }^{4}$ Semmelweis University, Department of Oncology, Budapest, Hungary
}

Radiol Oncol 2021; 55(2): 229-239.

Received 27 August 2020

Accepted 2 February 2021

Correspondence to: András Herein, National Institute of Oncology, Centre of Radiotherapy, Ráth György Street 7-9., H-1122 Budapest, Hungary. E-mail: hereina@gmail.com

Disclosure: No potential conflicts of interest were disclosed.

Background. The aim of the study was to dosimetrically compare multicatheter interstitial brachytherapy (MIBT) and stereotactic radiotherapy with CyberKnife (CK) for accelerated partial breast irradiation (APBI) especially concerning the dose of organs at risk (OAR-s).

Patients and methods. Treatment plans of thirty-two MIBT and CK patients were compared. The OAR-s included ipsilateral non-target and contralateral breast, ipsilateral and contralateral lung, skin, ribs, and heart for left-sided cases. The fractionation was identical $(4 \times 6.25 \mathrm{~Gy})$ in both treatment groups. The relative volumes (e.g. V100, V90) receiving a given relative dose $(100 \%, 90 \%)$, and the relative doses $\left(e . g . \mathrm{D} 0.1 \mathrm{~cm}^{3}, \mathrm{Dl} \mathrm{cm}^{3}\right)$ delivered to the most exposed small volumes $\left(0.1 \mathrm{~cm}^{3}, 1 \mathrm{~cm}^{3}\right)$ were calculated from dose-volume histograms. All dose values were related to the prescribed dose (25 Gy).

Results. Regarding non-target breast CK performed slightly better than MIBT (V100: $0.7 \%$ vs. 1.6\%, V50: $10.5 \%$ vs. $12.9 \%)$. The mean dose of the ipsilateral lung was the same for both techniques (4.9\%), but doses irradiated to volume of $1 \mathrm{~cm}^{3}$ were lower with MIBT (36.1\% vs. $\left.45.4 \%\right)$. Protection of skin and rib was better with MIBT. There were no significant differences between the dose-volume parameters of the heart, but with MIBT, slightly larger volumes were irradiated by $5 \%$ dose (V5: $29.9 \%$ vs. $21.2 \%$ ). Contralateral breast and lung received a somewhat higher dose with MIBT (Dlcm 3 : $2.6 \%$ vs. $1.8 \%$ and $3.6 \%$ vs. $2.5 \%)$.

Conclusions. The target volume can be properly irradiated by both techniques with similar dose distributions and high dose conformity. Regarding the dose to the non-target breast, heart, and contralateral organs the CK was superior, but the nearby organs (skin, ribs, ipsilateral lung) received less dose with MIBT. The observed dosimetric differences were small but significant in a few parameters at the examined patient number. More studies are needed to explore whether these dosimetric findings have clinical significance.

Key words: multicatheter interstitial brachytherapy; CyberKnife; APBI; dosimetric comparison 


\section{Introduction}

Breast radiotherapy after breast-conserving surgery reduces the risk of breast cancer recurrence by half and related mortality by one-sixth in earlystage breast cancer patients. ${ }^{1}$ Today, the accelerated partial breast irradiation (APBI) for treating early-stage breast cancer has been a widely accepted technique among radiation oncologists. ${ }^{2}$ APBI can be used because most of the local recurrences develop near the tumour bed requiring irradiation of the surroundings of the removed tumour, only. ${ }^{2}$ During APBI, a smaller volume needs to be irradiated compared to whole breast irradiation (WBI), so patients can tolerate an accelerated regimen of irradiation with higher fraction doses. In the case of APBI, the total treatment time is only 4-5 days instead of the few weeks of conventional WBI, and therefore, APBI is a preferred treatment option by patients. Because of the smaller irradiated volume, there are decreased doses to healthy tissues compared to WBI, which is another advantage of the APBI, and this is an important issue among long time survivors of breast cancer. ${ }^{3,4}$ In planning studies, and also in phantom measurements, it was verified that with smaller target volume the adjacent organs received less dose. 5,6

There are numerous techniques available for APBI. ${ }^{7}$ The first one was the multicatheter interstitial brachytherapy (MIBT), having the longest patient follow-up with excellent clinical results. ${ }^{8-15}$ Furthermore, recommendations for patient selection $^{16}$, target volume definition ${ }^{17,18}$ have been published, and practical guidelines are also available for $\mathrm{MIBT}^{19,20}$ ensuring appropriate quality assurance for this technique. Brachytherapy (BT) also performs well when it is used for boost irradiation following WBI.21,22 Other BT modalities have also been introduced, such as single/multichannel balloon therapy, non-invasive BT, and seed implantation. The external beam radiotherapy (EBRT) methods include 3D conformal radiotherapy (3D-CRT), intensity modulated radiotherapy (IMRT), volume modulated arc therapy (VMAT), CyberKnife therapy $(\mathrm{CK})$, proton therapy and intraoperative methods with electrons or $50 \mathrm{kV}$ photons. ${ }^{23}$

Dosimetric studies for APBI with $\mathrm{CK}$ are rare in the literature, and mainly focus on comparisons between different external beam techniques. CK dosimetry has been compared to 3D-CRT ${ }^{24-31}$, IMRT ${ }^{25,29,31}$, VMAT ${ }^{28,29}$, and tomotherapy. ${ }^{27} \mathrm{~A}$ dosimetric assessment of conformal and several different IMRT techniques for APBI is also available in the literature. ${ }^{32}$ Treatment plans of MIBT were also as- sessed against 3D-CRT ${ }^{33}$, IMRT ${ }^{34}$ and VMAT. ${ }^{35}$ Three years ago, a CK was installed at our institution, and very soon we started using it for APBI. Since we had lots of experience in interstitial BT we were interested in seeing how much the CK can imitate the BT, and whether it can be an alternative to BT.

Having surveyed the literature on APBI, we observed that the target volume can be irradiated properly with any technique, but differences exist in dosimetric issues of the organs at risk (OAR-s). In another study we compared plans of patients treated by CK with virtual MIBT plans based on CT images and contours of CK plans. ${ }^{36}$

The goal of this study was to dosimetrically compare the multicatheter interstitial brachytherapy and stereotactic irradiation with CyberKnife for APBI using separate patient cohorts with a special focus on dose to OAR-s. We believe that our results provide radiation oncologists and physicists with information on dosimetric advantages and disadvantages of these two image guided treatment techniques.

\section{Patients and methods}

Treatment plans of thirty-two MIBT patients and thirty-two CK patients consecutively treated at our institute between June 2017 and May 2020 were selected for a dosimetric comparison. Mostly, all patients were candidate for both techniques, and the treatment option was primarily chosen by the patient. All the patients were treated according to our institutional protocol, and in this dosimetric study we retrospectively collected and evaluated the treatment planning data, so no ethical approval was needed.

\section{Multicatheter interstitial brachytherapy}

The patients were treated with a high-doserate afterloader (microSelectron V3, Elekta, Brachytherapy, Veenendaal, The Netherlands) with Ir-192 stepping source. For patient selection and target volume definitions the GEC-ESTRO guidelines were followed. ${ }^{16,18}$ The number of implanted catheters ranged between 7 and 28, with a mean of 14. The catheters were placed into the breast with a template in a triangle pattern with $1.3 \mathrm{~cm}$ catheter separation, and the source step size was $2.5 \mathrm{~mm}$. The positions of the catheters were planned with image guidance using pre-implant CT imaging. ${ }^{37}$ The patients were treated according to plans made with an inverse planning method (Hybrid Planning 
Optimization, HIPO). For treatment planning the Oncentra Brachy v4.3 planning system (Elekta, Brachytherapy, Veenendaal, The Netherlands) was used. The calculation algorithm was based on the TG-43 formalism. ${ }^{38}$ After the catheter implantation, CT scans with a $3 \mathrm{~mm}$ slice distance were acquired. First, the lumpectomy cavity was outlined with the help of surgical clips placed into the cavity wall during the lumpectomy. To get the clinical target volume (CTV), a safety margin was added to the cavity according to the surgical tumour-free margin in all six main directions. The surgical and radiation margin together was always $20 \mathrm{~mm} .{ }^{18}$ The CTV was limited to skin, thoracic wall, and pectoral muscles. No additional margin was added to the CTV to create the planning target volume (PTV), so the PTV was always equal to the CTV. The outlined OAR-s were as follows: the ipsilateral and contralateral lungs and breasts, heart, skin, and ribs. The skin was defined as a $5 \mathrm{~mm}$ layer below the skin surface. The non-target ipsilateral breast was created from the ipsilateral breast and the PTV with a subtraction. After contouring all structures, the catheter reconstruction was carried out, and the dwell time optimization was performed. The source positions were activated within the PTV, and for selection an isodose line for prescription the aim was to achieve at least $90 \%$ target coverage by the prescribed dose (PD) while keeping the dose distribution relatively homogenous. A higher isodose line was selected if a proper dose homogeneity was maintained. Dosenonuniformity ratio (DNR) was used to measure the dose homogeneity. DNR is the ratio of volumes irradiated by 1.5 times of PD and PD $\left(\mathrm{V}_{1.5 \times \mathrm{PD}} / \mathrm{V}_{\mathrm{PD}}\right)$. The required value for DNR was less or equal to 0.35 . When coverage and homogeneity constraints could not be fulfilled at the same time, the target coverage was prioritized. For the OAR-s no dosevolume constraints were used, the parameters were only recorded. The PD was $25 \mathrm{~Gy}$ with a fractionation of $4 \times 6.25 \mathrm{~Gy}$, which is an accepted fractionation regime in the GEC-ESTRO VAPBI study. ${ }^{39}$ The treatment time of one fraction ranged between 5 and 15 minutes according to the volume of the PTV and the source strength.

\section{CyberKnife treatments}

The patients were treated with a CyberKnife M6 stereotactic system (Accuray, Sunnyvale, CA, USA) using InCise $2^{\mathrm{TM}}$ multileaf collimators (MLC-s) with step-and-shoot IMRT technique. ${ }^{40}$ The patient selection was the same as for MIBT. The mean monitor unit number was 2498.8 (range, 1578.7-4142.2), and the median segment number was 57 (range, 39-75). One week before the planning CT, four gold markers were implanted around the lumpectomy cavity with ultrasound guidance by an experienced interventional radiologist. The gold markers were used for real-time tracking during irradiation (Synchrony Respiration Tracking). Although surgical titanium clips were placed into the cavity wall during the lumpectomy, they do not provide enough contrast on X-ray images, and therefore they cannot be reliably tracked. That is why we used gold markers. The CT slice thickness for treatment planning was 1.25 $\mathrm{mm}$, and the images were acquired in exhalation breath-hold. The CT scans included both breasts and both sides of the lung. Metal artifact reduction CT scans were obtained to decrease the scattering effect of gold markers. The contouring protocol for CK was the same as for MIBT. However, to generate the PTV, a $2 \mathrm{~mm}$ margin was added to the CTV in each direction. With frequent intra-fraction imaging and correction by the robotic arm, the $2 \mathrm{~mm}$ margin seems to be enough to account for the nonrigid components of composite errors. ${ }^{41}$ For evaluation purpose, PTV_EVAL was created from PTV with the subtraction of the $5 \mathrm{~mm}$ skin layer. The prescribed dose was 25 Gy with fractionation of $4 x$ 6.25 Gy, exactly the same as in MIBT.

For planning, the Precision 1.1.1.1 (Accuray, Sunnyvale, CA, USA) software was used with a finite size pencil beam (FSPB) dose calculation algorithm for optimization and Monte Carlo algorithm with $0.5 \%$ uncertainty for plan evaluation. Regarding beam orientations, the contralateral breast and lung were allowed with "exit only" direction for optimization. Sequential optimization method was applied with the parameters of maximum $400 \mathrm{MU} /$ segments, $650 \mathrm{MU} /$ node, and for beam shaping all three types of segments (eroded, perimeter, random shape) were used with a 2.5 $\mathrm{mm}$ leaf margin. The aim was to achieve at least 99.5\% of V95 for PTV_EVAL and keep the maximum point dose under $120 \%$ of the PD. Regarding doses to OAR-s no constraints were used, only the orientation of the beams was optimized. Time reduction was used to reach the optimal treatment time without compromising the optimal dose distribution. The mean estimated treatment time was $33.3 \mathrm{~min}$ (range, $27-40 \mathrm{~min}$ ).

\section{Dosimetric evaluation and statistical analysis}

The treatment plan evaluation was performed using dose-volume histograms (DVH-s) (Figure 1). 


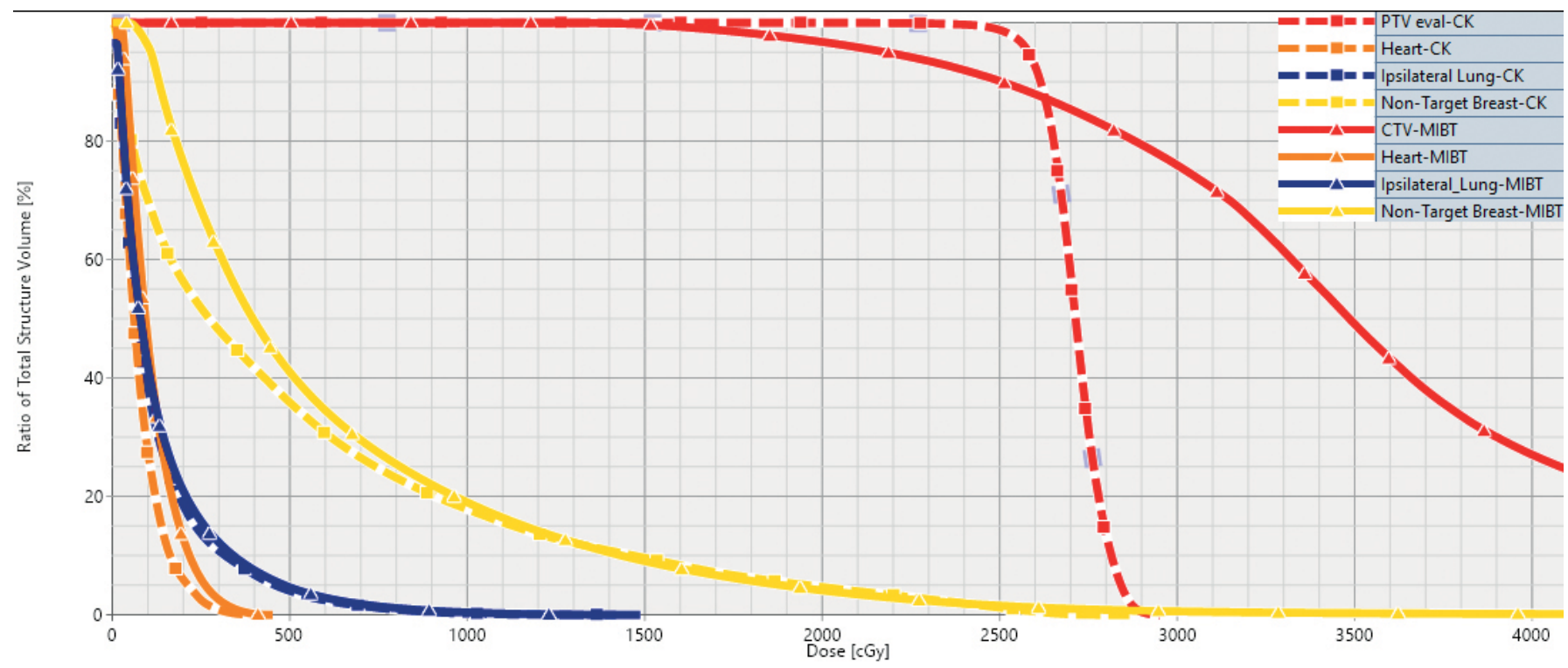

FIGURE 1. Representative dose-volume histograms (DVH-s) for multicatheter interstitial brachytherapy (MIBT) (solid line) and CyberKnife (CK) treatment (dashed line) for accelerated partial breast irradiation. The prescribed dose was 25 Gy. The target volume is indicated with red, the non-target breast with yellow, the ipsilateral lung with blue and the heart with orange. The solid red line clearly shows that with MIBT volumes irradiated by high doses (> 25 Gy) develop within the target volume.

All treatment plans and patient's data were anonymized for the dosimetric comparison. Quality parameters were derived as relative (e.g. V100, V90) or absolute volumes (e.g. V100 $\left(\mathrm{cm}^{3}\right)$, V50 $\left.\left(\mathrm{cm}^{3}\right)\right)$ receiving a percentage of the PD. Relative

TABLE 1. Tumour localization according to breast quadrants in patients treated by multicatheter interstitial brachytherapy (MIBT) and CyberKnife (CK)

\begin{tabular}{lcccccc}
\hline $\begin{array}{l}\text { Method of } \\
\text { treatment }\end{array}$ & Laterality & $\begin{array}{c}\text { Upper } \\
\text { outer }\end{array}$ & $\begin{array}{c}\text { Lower } \\
\text { outer }\end{array}$ & $\begin{array}{c}\text { Upper } \\
\text { inner }\end{array}$ & $\begin{array}{c}\text { Lower } \\
\text { inner }\end{array}$ & Central \\
\hline \multirow{2}{*}{ MIBT } & right & 3 & 1 & 3 & 2 & 4 \\
& left & 3 & 3 & 4 & 1 & 8 \\
CK & right & 6 & 6 & 2 & 1 & 2 \\
& left & 2 & 4 & 1 & 3 & 5 \\
\hline
\end{tabular}

TABLE 2. Absolute volumes of the body irradiated by $100 \%, 50 \%$ and $20 \%$ of the prescribed dose for multicatheter interstitial brachytherapy (MIBT) and CyberKnife (CK) treatments

\begin{tabular}{lccc}
\hline Body & MIBT & CK & p-value* \\
\hline $\mathrm{V} 100\left(\mathrm{~cm}^{3}\right)$ & $66.4(28.9-193.8)$ & $77.1(18.3-141.5)$ & 0.0356 \\
$\mathrm{~V} 50\left(\mathrm{~cm}^{3}\right)$ & $159.0(73.4-444.2)$ & $189.3(58.6-397.6)$ & 0.0184 \\
\hline 20 $\left(\mathrm{cm}^{3}\right)$ & $470.7(253.4-1078.1)$ & $609.1(326.7-1160.8)$ & 0.0013 \\
\hline
\end{tabular}

*Mann-Whitney U test doses in percentage of the PD to relative volumes (e.g. D5, D50) and to small absolute volumes (e.g. D0.1 $\mathrm{cm}^{3}, \mathrm{D} 1 \mathrm{~cm}^{3}$ ) were calculated, too. The conformity of dose distribution was characterized by COIN ${ }^{42}$ Most of these parameters are published in dosimetric studies what makes the intercomparison possible. Descriptive statistics were calculated to describe the treatment plans. Shapiro-Wilk W test was used on the distribution of dose-volume parameters to test normality. Since almost none of the parameters followed Gaussian distribution, the Mann-Whitney U test was used for every comparison with the Statistica 10.0 software (StatSoft, Inc., Tulsa, OK, USA) and $\mathrm{p}<0.05$ was considered statistically significant.

\section{Results}

Thirteen MIBT patients (41\%) had right-sided and nineteen (59\%) left-sided tumours, and seventeen CK patients $(53 \%)$ had right-sided and fifteen (47\%) left-sided lesions. The distribution of tumour localization is shown in Table 1.

The mean volume of the ipsilateral breast was $825.7 \mathrm{~cm}^{3}$ (range, 386.9-2097.5 $\mathrm{cm}^{3}$ ) and $984.4 \mathrm{~cm}^{3}$ (range, 372.7-2437.1 $\mathrm{cm}^{3}$ ) for the MIBT and CK patients, respectively $(\mathrm{p}=0.4400)$. The mean volume of the PTV was $58.2 \mathrm{~cm}^{3}$ (range, $26.6-173.6 \mathrm{~cm}^{3}$ ) for 
MIBT patients and $71.7 \mathrm{~cm}^{3}$ (range, 17.1-129.1 $\mathrm{cm}^{3}$ ) for CK patients, which was significantly higher $(p$ $=0.0101)$. The mean volume of the CTV was 51.9 $\mathrm{cm}^{3}$ (range, 10.2-96.4 $\mathrm{cm}^{3}$ ) for CK, which did not differ significantly from the volume of the CTV for MIBT $\left(58.2 \mathrm{~cm}^{3}, \mathrm{p}=0.8456\right)$. For MIBT the V100 for PTV was 91.4\% (range, 83.1-96.4\%) meanwhile the DNR was 0.35 (range, 0.24-0.44). For PTV_EVAL of CK patients, the V100 and V95 were $97.5 \%$ (range, 95.2-99.3\%) and $99.7 \%$ (range, $97.7-100 \%$ ), respectively, and the mean of the maximum doses was $116.5 \%$ (range, $113.6-125 \%$ ). Because of the smaller target volumes, the V100, V50, and the V20 absolute volume parameters calculated for the whole body were significantly smaller for MIBT than for CK as it is presented in Table 2.

But, by relating these mean volumes to mean PTV volumes, it can be observed that the CK irradiated relatively less normal tissues, especially at higher dose levels. For MIBT and CK, the ratio of $\mathrm{V} 100_{\text {BODY }} / \mathrm{V}_{\text {PTV }}$ is 1.14 vs. 1.08 , and for $\mathrm{V} 50_{\text {BODY }} / \mathrm{V}_{\text {PTV }}$ it is 2.73 vs. 2.64. Only the $\mathrm{V} 20_{\text {BODY }} / \mathrm{V}_{\text {PTV }}$ is smaller for MIBT (8.09 vs. 8.5).

The conformity of the dose distributions was very high for both techniques (Figure 2), and the dosimetric parameters for ipsilateral breast were very similar (Table 3). Only the PD irradiated significantly less volume of the non-target breast at CK $(0.7 \%$ vs. $1.6 \%, \mathrm{p}<0.0001)$. For lower dose values, the CK resulted in slightly smaller volumes, but without statistical significance. Large conformity indices (COIN-s) demonstrate the high dose conformity for both techniques. The COIN was 0.74 for MIBT and 0.88 for CK patients $(\mathrm{p}<0.001)$.

The mean dose of the ipsilateral lung was exactly the same (4.9\%) for both techniques (Table 4). All other parameters were smaller for MIBT, and the doses to the most exposed low volumes $\left(0.1 \mathrm{~cm}^{3}, 1\right.$ $\mathrm{cm}^{3}$ ) were significantly less.

Heart dosimetry was examined only for left-sided cases. The results are shown in Table 5. For each parameter, larger values were obtained with MIBT, but none of the differences was significant.

The protection of skin and ribs was better with MIBT. The differences were significant for all parameters, as shown in Table 6 . The maximal dose to the ribs never exceeded the PD in MIBT cases $\left(\mathrm{V} 100\left(\mathrm{~cm}^{3}\right)=0\right)$. The mean value for $\mathrm{V} 100\left(\mathrm{~cm}^{3}\right)$ in CK patients was small, only $0.2 \mathrm{~cm}^{3}$, and the maximal dose exceeded the PD in 11 cases.

For contralateral breast and lung, volumetric dose parameters were very low for both techniques, but CK resulted in significantly lower values in each parameter, as it is shown in Table 7.

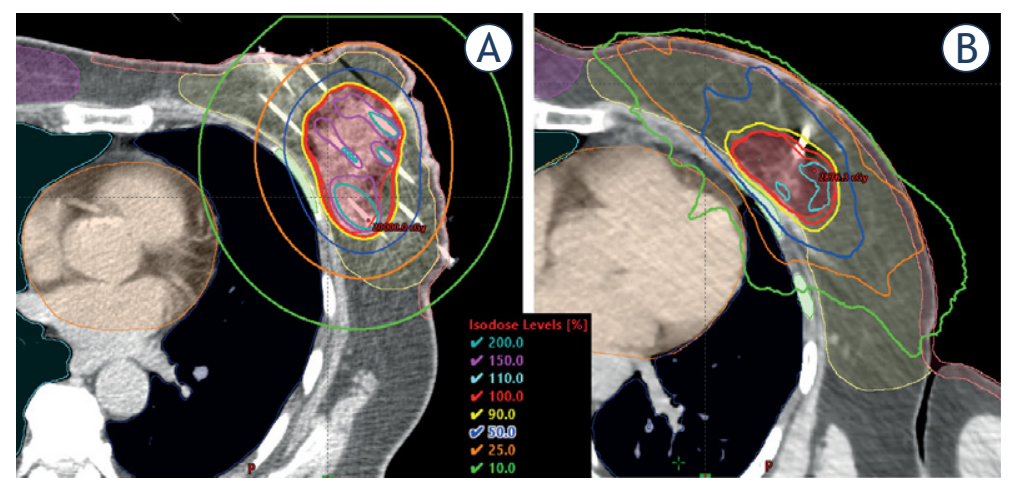

FIGURE 2. Representative dose distribution for multicatheter interstitial brachytherapy (MIBT) (A) and CyberKnife (CK) treatment (B). PTV: red, ipsilateral breast: yellow, contralateral breast: pink, ribs: green, heart: orange, ipsilateral lung: dark blue, contralateral lung: light blue.

\section{Discussion}

As a result of the technological development during the last decade, a number of new techniques have been introduced for APBI, and the question

TABLE 3. Dosimetry of ipsilateral breast for multicatheter interstitial brachytherapy (MIBT) and Cyberknife (CK) treatments. The values are given in percentages

\begin{tabular}{lccc}
\hline \multicolumn{1}{c}{ MIBT } & CK & p-value* \\
\hline Whole breast & & & \\
\hline V100 & $9.7(2.2-29.7)$ & $9.6(0.9-20.4)$ & 0.9381 \\
V50 & $23.2(5.1-68.0)$ & $23.3(2.7-47.1)$ & 0.9822 \\
\hline Non-target breast & & & \\
\hline V100 & $1.6(0.4-4.2)$ & $0.7(0.0-2.0)$ & $<0.0001$ \\
V90 & $2.8(0.6-8.1)$ & $2.3(0.0-4.4)$ & 0.7019 \\
V50 & $12.9(3.1-35.4)$ & $10.5(1.7-18.9)$ & 0.5501 \\
V25 & $31.8(9.4-68.4)$ & $29.9(7.6-55.2)$ & 0.8772 \\
\hline
\end{tabular}

*Mann-Whitney U test

TABLE 4. Dosimetry of ipsilateral lung for multicatheter interstitial brachytherapy (MIBT) and Cyberknife (CK) treatments. The values are given in percentages

\begin{tabular}{lccc}
\hline Ipsilateral lung & MIBT & CK & p-value* \\
\hline Mean lung dose & $4.9(1.9-11.1)$ & $4.9(1.6-11.8)$ & 0.9946 \\
D0.1 cm & $41.0(10.1-61.5)$ & $52.2(15.6-90.5)$ & 0.0068 \\
\hline $\mathrm{D} 1 \mathrm{~cm}^{3}$ & $36.1(8.7-55.8)$ & $45.4(14.4-84.5)$ & 0.0162 \\
$\mathrm{D} 2 \mathrm{~cm}^{3}$ & $33.6(8-52.5)$ & $42.2(13.7-80.5)$ & 0.0198 \\
\hline $\mathrm{D} 2$ & $21.2(7.4-32.9)$ & $24(8.8-46.4)$ & 0.2454 \\
V5 & $30.6(5.1-50.0)$ & $30.7(5.8-63.2)$ & 0.9357 \\
\hline
\end{tabular}

*Mann-Whitney U tes† 
now arises whether which technique is the most advantageous for the patients. Based on large trials, at selected patients, the non-inferiority of APBI against WBI was confirmed, but between the various APBI techniques, only minor differences exist. ${ }^{11}$ Dosimetric analysis can explore these differences providing some guidance for clinicians in selecting the appropriate method for a patient. Papers have been published on dosimetric comparisons between different APBI techniques ${ }^{24,43-46}$; and a few papers are available about the comparison of CyberKnife and other external beam techniques. ${ }^{26-29}$ However, to our knowledge, this analysis is the first one which compares the dosimetric differences between the multicatheter interstitial brachytherapy and CyberKnife treatments for partial breast irradiation using clinical plans.

A couple of studies have investigated the feasibility of APBI with CK. The treatment characteristics with the dosimetric data from the literature are shown in Table 8. In most of the studies, cones or the Iris applicator were used, and the most frequent fractionation scheme was 5 x $6 \mathrm{~Gy}$, but clinical results of dose escalation up to $5 \times 8 \mathrm{~Gy}$ are also available now. ${ }^{47}$ In the studies, the CTVPTV margin ranged between 0 and $5 \mathrm{~mm}$. With the introduction of the MLC, the treatment time remarkably decreased, compared to using cones or the Iris applicator. It is difficult to make quantitative dosimetric comparisons between studies because the reported parameters are too diverse. The first report on using stereotactic radiotherapy for breast tumours was published in 2009.48 The study showed the feasibility of irradiation with $\mathrm{CK}$, but dosimetric details were not reported. The earliest study with dosimetry was performed by Vermeulen et al. ${ }^{49}$, and based on their results, the authors recommend the $\mathrm{CK}$ as a suitable treatment method for APBI.

They compared their dosimetric data with the constraints of 3D-CRT used in the NSABP-39/ RTOG 0413 protocol. ${ }^{50}$ On average, $11 \%$ of the ipsilateral breast received the PD (V100), and 23\% (for two patients) and $26 \%$ (for seven patients) were irradiated by the half of the PD (V50). These values are well below the constraints of the NSABP-39/ RTOG 0413 protocol and are in good agreement with our $\mathrm{CK}$ results (V100 $=9.6 \%, \mathrm{~V} 50=23.3 \%)$. We note that for MIBT patients, we obtained practically the same values (V100 $=9.7 \%$, V50 = 23.2\%). In 2014, the same group published dosimetric data of twenty-one patients, but those basically did not differ from the previous ones. ${ }^{51}$ In a retrospective planning study published by $\mathrm{Xu}$ et al. ${ }^{25}$ for fourteen patients previously treated with 3D-CRT, additional CK plans were created and compared with 3D-CRT and IMRT plans. Since in CK plans very homogenous dose distributions were created, the mean V100 parameter was $95.7 \%$, which is lower than the value in our study $(97.5 \%)$. The maximum dose in PTV was kept on 104\% which is much lower than presented in this study $(116.5 \%)$. The mean V100 and V50 parameters of the uninvolved normal breast were $9 \%$ and $23.1 \%$, which are much higher than our values ( $0.7 \%$ and $10.5 \%)$. Probably, because of the relatively homogenous dose distribution, the dose gradient was not steep enough to achieve very low V100 parameters for the breast. Another explanation for our better dose conformity can be the use of MLC-s with step-and-shoot IMRT technique. For the median V5 parameter of ipsilateral lung, their analysis resulted in 31.4\%, which is very similar to our $30.7 \%$. In another retrospective study, Obayomi et al. ${ }^{52}$ analyzed the dosimetric results of $10 \mathrm{CK}$ treated patients. The mean PTV volume was $70 \mathrm{~cm}^{3}$, very similar to ours (71.7 $\mathrm{cm}^{3}$ ). The fractionation was 5 x $6 \mathrm{~Gy}(30 \mathrm{~Gy})$, and the authors reported the maximum doses of skin and ribs as $32 \mathrm{~Gy}(107 \%$ of PD) and $26 \mathrm{~Gy}(87 \%$ of PD), consecutively. In our assessment, for skin and ribs, the D0.1 $\mathrm{cm}^{3}$ value was $88.8 \%$ and $78.9 \%$. In the paper of Lozza et al. ${ }^{53}$, mainly the clinical results from $20 \mathrm{CK}$ treated patients were reported. Among 20 patients, the mean volume of PTV was $88.1 \mathrm{~cm}^{3}$, and the V5 value of heart for left-sided cases was $14.0 \%$, which is lower than in our study $(21.2 \%)$. For skin, they examined the Dmax parameter, which was $98.2 \%$ of the PD. In our study, the D0.1 $\mathrm{cm}^{3}$ was $88.8 \%$. In a recent paper, Lee et al..$^{54}$ published their first experience in Korea of partial breast irradiation with CK. In their study, on average, $35.5 \%$ of ipsilateral breast volume received $50 \%$ of the PD, while in our case, only $23.3 \%$. The mean volume of PTV was similar $\left(73.6 \mathrm{~cm}^{3}\right.$ vs. 71.7 $\mathrm{cm}^{3}$ ), although they did not use any CTV-PTV margin. But, the ipsilateral breast volume of their patients was less than half compared to ours (481.1 $\mathrm{cm}^{3}$ vs. $984.4 \mathrm{~cm}^{3}$ ). Despite the fact, that Korean women have smaller breast the authors consider the CK technique as a feasible method for APBI. They found that the mean maximal skin dose was $89 \%$, while in our case, the D0.1 $\mathrm{cm}^{3}$ was $89 \%$. The mean dose to heart for left-sided lesions was also similar $(2.3 \%$ vs. $3.3 \%)$.

APBI treatment with CyberKnife is feasible and compared to other external beam techniques the CK plans produce better dosimetric parameters regarding the OAR-s. Xu et al. ${ }^{25}$ found $\mathrm{CK}$ plans more 
conformal and observed better dose sparing of the critical structure except for the extremely low dose region (for example, V1 for ipsilateral lung) compared to IMRT and 3D-CRT. In a quantitative analysis with ten patients, Lee at al. ${ }^{29}$ compared CK with 3D-CRT, IMRT and VMAT for APBI. They only examined patients with left-sided lesions and had a very similar conclusion about conformity of dose distribution as Xu et al. $25 .{ }^{25}$ Rault et al. ${ }^{27}$ examined the dosimetric effect of respiratory tracking at CK treatments against Tomotherapy and 3D-CRT. The authors stated that the non-coplanarity of the CK resulted in significantly less dose to the normal tissue of ipsilateral breast, in case of the $2 \mathrm{~mm}$ CTV-PTV margin. A very profound analysis was performed by Goggin et al. ${ }^{26}$ in comparing different CK treatment plans (Iris, MLC) with 3D-CRT ones. Based on the analysis of nine patients, they found that CK-MLC technique resulted in lower dose to the ipsilateral lung than CK-Iris and comparable treatment time with 3D-CRT. In an examination with ten patients, Bonfantini et al..$^{28}$ compared CK with 3D-CRT and VMAT. Based on their analysis, 3D-CRT gives a reduction of the dose to OAR-s except for ipsilateral breast.

Major et al. ${ }^{34}$ reported results of MIBT against co-planar IMRT. They found that MIBT generally spares normal tissues and organs at risk (except for heart) better than IMRT. They found significant differences in non-target breast dosimetry parameters in favour of MIBT. Inverse planning can improve the plan quality of breast implants, better homogeneity and a few percent less dose to OAR-s can be achieved at identical target coverage. ${ }^{55,56}$ In this way we made a comparison between the treatment plans of the most advanced inverse optimized BT and a high-tech stereotactic EBRT technique.

In a recent publication our group reported a comparative dosimetry between CK and MIBT, but in that work ideal implant geometry was assumed in the hypothetical BT plans. ${ }^{36}$ Therefore, the strength of the conclusion is weaker than in case of comparison between real clinical plans. Anyway, the findings regarding the dose to OAR-s are very similar to the ones observed in current study.

Comparing the dose distributions between MIBT and EBRT techniques, the CK seems most similar to the MIBT regarding the shapes of the isodose lines between $50-100 \%$ of PD. The explanation can be the use of lots of non-isocentric beams entering from different directions, not limited from one plane, which results in more focused 3D dose delivery to the PTV. This is presented in Figure 2, where the isodose lines between $50-100 \%$ of PD following the shape of the PTV are very similar for both techniques. But, differences exist because volumes irradiated by high doses always develop in BT, which is shown in Figure 2 by the 150 and $200 \%$ isodose lines. However, the low isodoses around BT implant become nearly circular, while in CK case, they are irregular due to the multiple

TABLE 5. Dosimetry of heart for multicatheter interstitial brachytherapy (MIBT) and CyberKnife (CK) treatments. The values are given in percentages

\begin{tabular}{lccc}
\hline Heart & MIBT & CK & p-value* \\
\hline Mean heart dose & $4.1(1.0-7.7)$ & $3.3(1.3-6.9)$ & 0.1706 \\
D0.1 cm & $21.4(4.0-47.2)$ & $18.9(8.4-43.2)$ & 0.2822 \\
\hline $\mathrm{D} 1 \mathrm{~cm}^{3}$ & $18.2(3.2-42.3)$ & $16.6(7.3-40.3)$ & 0.4878 \\
$\mathrm{D} 2 \mathrm{~cm}^{3}$ & $17(3.0-40.0)$ & $15.6(6.8-38.3)$ & 0.5324 \\
$\mathrm{D} 2$ & $13.3(4.3-26.4)$ & $12.5(5.2-31.5)$ & 0.5098 \\
$\mathrm{~V} 5$ & $29.9(0.0-62.5)$ & $21.2(6.1-41.0)$ & 0.1653 \\
\hline
\end{tabular}

*Mann-Whitney U test

TABLE 6. Dosimetry of skin and ribs for multicatheter interstitial brachytherapy (MIBT) and Cyberknife (CK) treatments. The values are given in percentages except for $\vee 100$ and $\vee 50$

\begin{tabular}{|c|c|c|c|}
\hline Skin & MIBT & CK & p-value* \\
\hline $\mathrm{D} 0.1 \mathrm{~cm}^{3}$ & $75.3(18.0-164.2)$ & $88.8(27.6-112.4)$ & 0.0080 \\
\hline $\mathrm{D} 0.2 \mathrm{~cm}^{3}$ & 70.3 (17.4-140.7) & $86.8(21.1-111.1)$ & 0.0053 \\
\hline $\mathrm{Dlcm} \mathrm{cm}^{3}$ & $58.4(15.0-97.1)$ & $79(22.8-106.0)$ & 0.0007 \\
\hline \multicolumn{4}{|l|}{ Ribs } \\
\hline $\mathrm{D} 0.1 \mathrm{~cm}^{3}$ & 53.7 (8.1-92.7) & $78.9(17.6-108.4)$ & 0.0003 \\
\hline $\mathrm{Dlcm} \mathrm{cm}^{3}$ & $43.1(5.3-76.4)$ & $69.1(16.4-105)$ & 0.0004 \\
\hline V100 $\left(\mathrm{cm}^{3}\right)$ & $0(0-0)$ & $0.2(0.0-3.0)$ & 0.0101 \\
\hline V50 $\left(\mathrm{cm}^{3}\right)$ & $2(0.0-16.4)$ & $4.2(0.0-13.4)$ & 0.0105 \\
\hline
\end{tabular}

*Mann-Whitney U test

TABLE 7. Dosimetry of contralateral breast and lung for multicatheter interstitial brachytherapy (MIBT) and Cyberknife (CK) treatments. The values are given in percentages

\begin{tabular}{lccc}
\hline $\begin{array}{l}\text { Contralateral } \\
\text { breast }\end{array}$ & MIBT & CK & p-value* \\
\hline D0.1 cm & $4(0.0-9.8)$ & $2.1(0.3-11.2)$ & 0.0039 \\
\hline 1 $\mathrm{cm}^{3}$ & $2.6(0.0-6.4)$ & $1.8(0.3-9.9)$ & 0.0378 \\
\hline Contralateral lung & & & \\
\hline$D 0.1 \mathrm{~cm}^{3}$ & $5.5(0.0-11.7)$ & $3(1.0-5.6)$ & 0.0000 \\
\hline $\mathrm{D} 1 \mathrm{~cm}^{3}$ & $3.6(0.0-8.3)$ & $2.5(0.6-4.8)$ & 0.0228 \\
\hline
\end{tabular}

*Mann-Whitney U test 
TABLE 8. Treatment characteristics and mean dosimetric parameters in publications for accelerated partial breast irradiation (APBI) with CyberKnife

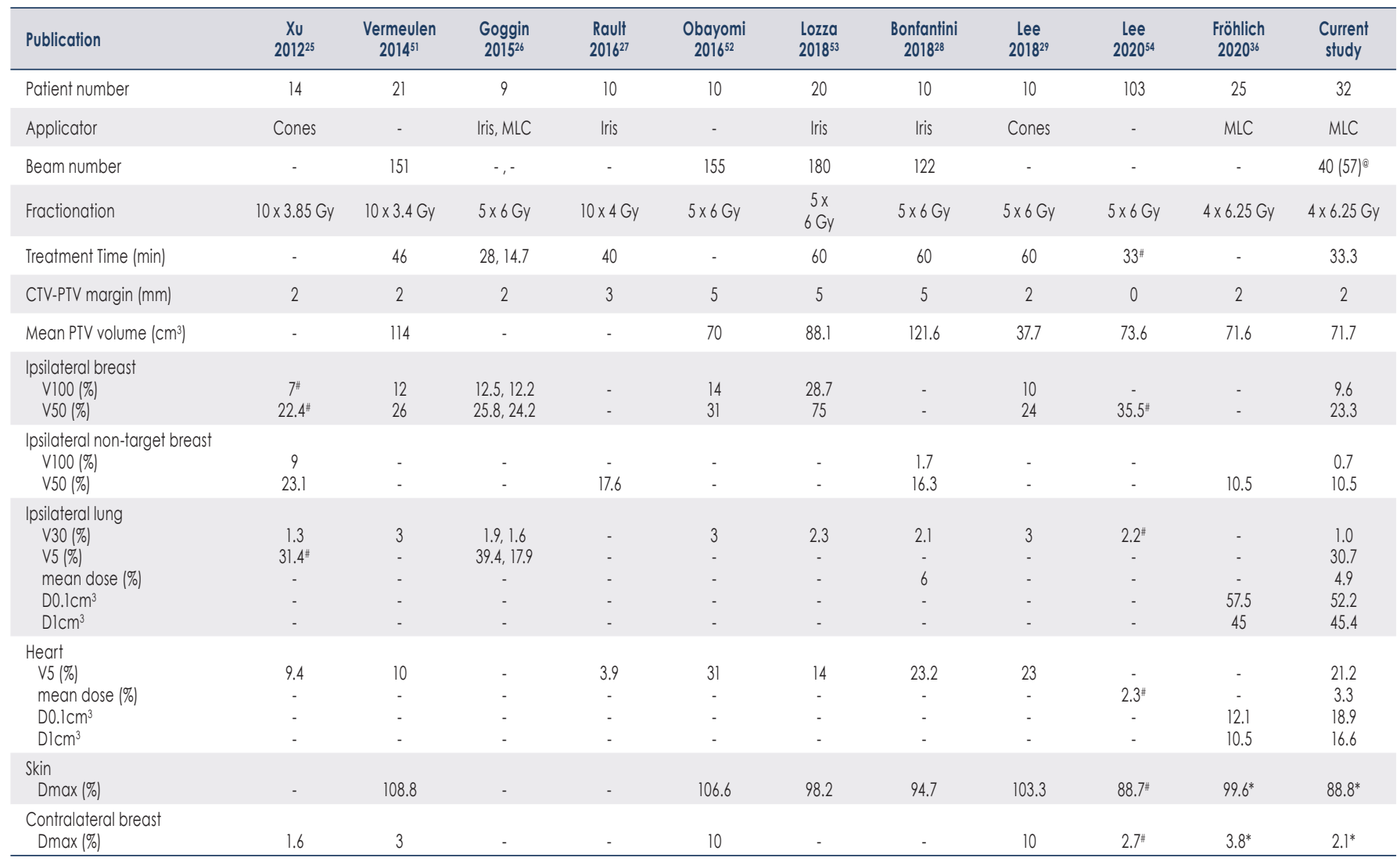

* = D0.1 $\mathrm{cm}^{3} ; \#=$ median value; ${ }^{\circledR}=$ segment number in parentheses; CTV = clinical target volume; MLC = multileaf collimator; PTV = planning target volume

non-coplanar beam entries. The absolute volumes encompassed by $100 \%, 50 \%$, and $20 \%$ isodose surfaces are $16 \%, 19 \%$, and $29 \%$ larger at CK compared to MIBT (Table 2). The volume of the ipsilateral breast was slightly larger in the CK group (984.4 $\mathrm{cm}^{3}$ vs. $\left.825.7 \mathrm{~cm}^{3}\right)$, but the PTV was also bigger $(71.7$ $\mathrm{cm}^{3}$ vs. $58.2 \mathrm{~cm}^{3}$ ), and this explains why the relative volumes of ipsilateral breast irradiated by the $100 \%$ and $50 \%$ dose for the two techniques were nearly identical (Table 3). There was no difference between the mean lung doses and the volumes irradiated by low dose (V5) for the two techniques (Table 4). However, the other examined dose parameters were significantly smaller with MIBT (D0.1 $\mathrm{cm}^{3}: 41.0 \%$ vs. $52.2 \%$, D $1 \mathrm{~cm}^{3}: 36.1 \%$ vs. $45.4 \%$ ). The heart was better protected with $\mathrm{CK}$, since all dosimetric parameters were smaller, but without statistical significance (Table 5). This observation is similar to the findings of Major et al. ${ }^{34}$ when MIBT was compared to IMRT. In our CK group, almost half of the tumours located in one of the upper quadrants, and in these cases, the heart protection was better maintained because the beams could avoid the heart directly. In most cases, the skin and ribs are close to the PTV, sometimes they are contacting, and that is why the MIBT performs better for these two organs. In close proximity to the implant, due to inverse square law, the dose gradient is high resulting in proper protection of adjoining organs. In our study, all dose parameters for skin and ribs were significantly lower for MIBT than for CK (Table 6). We note that the TG43 formalism overestimates the skin dose because it assumes a full scattering condition, which is not fulfilled at all in breast BT. ${ }^{34,57}$ From this follows that the real differences are even higher in favour of MIBT. The contralateral breast and lung receive low doses at both techniques, but the values were smaller with CK (Table 7). These organs are usually far from the PTV, and therefore in MIBT there is no way to lessen the dose without compromising the target coverage. In contrast, with proper selection 
of beam orientations, such as choosing 'exit only' option for these volumes at optimization, the dose can be kept lower at CK. Both techniques require invasive intervention, but with different degrees of invasiveness. At MIBT sometimes quite a lot of catheters are implanted, but at CK the gold markers are inserted with 2-3 needles, only. The overall treatment times of CK and MIBT treatments were similar, every patient finished the therapy in four days.

The limitation of our study is that the dosimetric evaluation was performed on two separate patient populations. However, the patient selection, target volume definition, and organ delineations in the two patient cohorts were performed using the same rules by three experienced radiotherapists providing appropriate consistency. The volumes of ipsilateral breasts and CTV-s were similar in the two groups meaning a proper balance with respect to base-line dosimetry. The differences in patient characteristics and individual anatomy in two patient cohorts may cause some uncertainties regarding dosimetric comparison. However, this serves the purpose of assessment of real treatments. Using the same CT-data with identical contours to compare the two treatment techniques would decrease the uncertainties, but in that case real treatments are compared with hypothetical ones which can never be realized..$^{36}$ In contrast, the advantage of our current analysis is that both investigated treatment methods reflect reality, as in the study of Weed et al. ${ }^{24}$, in which MIBT against MammoSite ${ }^{\mathrm{TM}}$ and 3D-CRT techniques was compared for three different patient cohorts. Our investigated two techniques are routinely used in our clinical practice, therefore the obtained results are realistic. However, whether the observed small dosimetric differences translate into different clinical outcomes requires further investigations with an assessment of more data.

\section{Conclusions}

In APBI, the dose distributions of MIBT and CK are similar with high dose conformity and comparable isodose lines between $50-100 \%$ of PD, but at MIBT regions in the target volume irradiated by very high doses develop due to unique feature of the BT. The low dose isodose lines are nearly circular at MIBT and irregular at CK cases. Regarding the PD the CK treatments mimic well the BT irradiation with taking advantage of non-coplanar beam entries and management of organ motion. OAR-s close to tumour bed (skin, ribs, ipsilateral lung) can be better protected with MIBT, but CK performs better for other organs (heart, contralateral breast, and lung). The advantage of CK is its less invasiveness, but its accessibility is very limited. BT is an easily accessible technique in almost all radiotherapy departments, but it demands proper skills with good dexterity.

\section{Acknowledgements}

This study was supported by the Hungarian Thematic Excellence Programme (TKP2020NKA-26).

\section{References}

1. Darby S, Mc Gale P, Correa C, Taylor C, Arriagada R, Clarke M, et al; Early Breast Cancer Trialists' Collaborative Group (EBCTCG). Effect of radiotherapy after breast-conserving surgery on 10-year recurrence and 15-year breast cancer death: meta-analysis on individual patient data for 10,801 women in 17 randomised trials. Lancet 2011; 378: 1707-16. doi: 10.1016/S01406736(11)61629-2

2. Bennion NR, Baine M, Granatowicz A, Wahl AO. Accelerated partial breast radiotherapy: a review of the literature and future directions. Gland Surg 2018; 7: 596-610. doi: 10.21037/gs.2018.11.05

3. Grantzau T, Overgaard J. Risk of second non-breast cancer after radiotherapy for breast cancer: a systematic review and meta-analysis of 762,468 patients. Radiother Oncol 2015; 114: 56-65. doi:10.1016/j.radonc.2014.10.004

4. Marcu LG, Santos A, Bezak E. Risk of second primary cancer after breast cancer treatment. Eur J Cancer Care 2014; 23: 51-64. doi: 10.1111/ecc.12109

5. Lara TRM, Fleury E, Mashouf S, Helou J, McCann C, Ruschin M, et al. Measurement of mean cardiac dose for various breast irradiation techniques and corresponding risk of major cardiovascular event. Front Oncol 2014; 4: 284. doi: 10.3389/fonc.2014.00284

6. Duma MN, Baumann R, Budach W, Dunst J, Feyer P, Fietkau R, et al. Heartsparing radiotherapy techniques in breast cancer patients: a recommendation of the breast cancer expert panel of the German Society of Radiation Oncology (DEGRO). Strahlenther Onkol 2019; 195: 861-71. doi: 10.1007/ s00066-019-01495-w

7. Forster T, Köhler CVK, DebusJ, Hörner-Rieber J. Accelerated partial breast irradiation: a new standard of care? Breast Care 2020; 15: 136-47. doi: 10.1159/000506254

8. Polgár Cs, Major T, Fodor J Sulyok Z, Somogyi A, Lövey K, et al. Accelerated partial breast irradiation using high-dose-rate interstitial brachytherapy: 12-year update of a prospective clinical study. Radiother Oncol 2010; 94: 274-79. doi: 10.1016/j.radonc.2010.01.019

9. Polgár Cs, Fodor J, Major T, Sulyok Z, Kásler M. Breast-conserving therapy with partial or whole breast irradiation: ten-year results of the Budapest randomized trial. Radiother Oncol 2013; 108: 197-202. doi: 10.1016/j. radonc.2013.05.008

10. Polgár Cs, Ott OJ, Hildebrant G, Kauer-Dorner D, Knauerhase H, Major $T$ et al. Late side-effects and cosmetic results of accelerated partial breast irradiation with interstitial brachytherapy versus whole-breast irradiation after breast-conserving surgery for low-risk invasive and in-situ carcinoma of the female breast: 5-year results of a randomised, controlled, phase 3 trial. Lancet Oncol 2017; 18: 259-68. doi: 10.1016/S1470-2045(17)30011-6

11. Strnad V, Ott OJ, Hildebrandt G, Kauer-Dorner D, Knauerhase H, Major T, et al. 5-year results of accelerated partial breast irradiation using sole interstitial multicatheter brachytherapy versus whole-breast irradiation with boost after breast-conserving surgery for low-risk invasive and in-situ carcinoma of the female breast: a randomized, phase 3, non-inferiority trial. Lancet 2016; 387: 229-38. doi: 10.1016/S0140-6736(15)00471-7 
12. Ott OJ, Strnad V, Hildebrant G, Kauer-Dorner D, Knauerhase H, Major T, et al GEC-ESTRO multicenter phase 3-trial: accelerated partial breast irradiation with interstitial multicatheter brachytherapy versus external beam whole breast irradiation: early toxicity and patient compliance. Radiother Oncol 2016; 120: 119-23. doi: 10.1016/j.radonc.2016.06.019

13. Soror T, Kovács G, Seibold N, Melchert C, Baumann K, Wenzel E, et al. Cosmetic changes following surgery and accelerated partial breast irradiation using HDR interstitial brachytherapy. Strahlenther Onkol 2017; 193 367-74. doi: 10.1007/s00066-016-1093-6

14. Schafer R, Strnad V, Polgár Cs, Uter W, Hildebrandt G, Ott OJ, et al. Quality-oflife results for accelerated partial breast irradiation with interstitial brachy therapy versus whole-breast irradiation in early breast cancer after breast conserving surgery (GEC-ESTRO): 5-year results of a randomised, phase 3 trial. Lancet Oncol 2018; 19: 834-44. doi: 10.1016/S1470-2045(18)30195-5

15. Vicini F, Cecchini R, White J, Arthur DW, Julian TB, Rabinovitch RA, et al. Long-term primary results of accelerated partial breast irradiation afte breast-conserving surgery for early-stage breast cancer: a randomised phase 3, equivalence trial. Lancet 2019; 394: 2155-64. doi: 10.1016/S01406736(19)32514-0

16. Polgár Cs, van Limbergen E, Pötter R, Kovács Gy, Polo A, Lyczek J, et al. Patient selection for accelerated partial breast irradiation (APBI) after breast conserving surgery: recommendations of the Groupe Européen de Curiethérapie-European Society for Therapeutic Radiology and Oncology (GEC-ESTRO) Breast Cancer Working Group based on clinical evidence (2009). Radiother Oncol 2010; 94: 264-73. doi: 10.1016/j.radonc.2010.01.014

17. Strnad V, Hannoun-Levi JM, Guinot JL, Lössl K, Kauer-Dorner D, Resch A et al. Recommendations from GEC ESTRO breast cancer working group (I): target definition and target delineation for accelerated or boost partial breast irradiation using multicatheter interstitial brachytherapy after breast conserving closed cavity surgery. Radiother Oncol 2015; 115: 342-8. doi: 10.1016/j.radonc.2015.06.010

18. Major T, Gutiérrez C, Guix B, Van Limbergen E, Strnad V, Polgár Cs, et al; on behalf of Breast Cancer Working Group of GEC-ESTRO. Recommendations from GEC ESTRO Breast Cancer Working Group (II): target definition and target delineation for accelerated or boost partial breast irradiation using multicatheter interstitial brachytherapy after breast conserving open cavity surgery. Radiother Oncol 2016; 18: 199-204. doi: 10.1016/j.radonc.2015.12.006

19. Strnad V, Major T, Polgár Cs, Lotter M, Guinot JL, Gutierrez-Miguelez C, et al. ESTRO-ACROP guideline: interstitial multi-catheter breast brachytherapy as accelerated partial breast irradiation alone or as boost - GEC-ESTRO breast cancer working group practical recommendations. Radiother Oncol 2018; 128: 411-20. doi: 10.1016/j.radonc.2018.04.009

20. Strnad V, Krug D, Sedlmayer F, Piroth MD, Budach W, Baumann R, et al DEGRO practical guideline for partial-breast irradiation. Strahlenther Onkol 2020; 196: 749-63. doi: 10.1007/s00066-020-01613-z

21. Kindts I, Laenen A, Christiansen M, Janssen H, Van Limbergen E, Weltens C. Comparison of brachytherapy and external beam radiotherapy boost in breast-conserving therapy: patient-reported outcome measures and aesthetic outcome. Strahlenther Onkol 2019; 195: 21-31. doi: 10.1007/ s00066-018-1346-7

22. Kindts I, Verhoeven $\mathrm{K}$, Laenen $\mathrm{A}$, Christiaens $\mathrm{M}$, Janssen $\mathrm{H}$, Van der Vorst $\mathrm{A}$ et al. A comparison of a brachytherapy and an external beam radiotherapy boost in breast-conserving therapy for breast cancer: local and any recurrences. Strahlenther Onkol 2019; 195; 310-7. doi: 10.1007/s00066-018$1413-0$

23. Njeh CF, Saunders MW, Langton CM. Accelerated partial breast irradiation (APBI): a review of available techniques. Radiat Oncol 2010; 5: 90 . doi: 10.1186/1748-717X-5-90

24. Weed DW, Edmundson GK, Vicini FA, Chen PY, Martinez AA. Accelerated partial breast irradiation: a dosimetric comparison of three different techniques. Brachytherapy 2005; 4: 121-9. doi: 10.1016/j.brachy.2004.12.005

25. Xu Q, Chen Y, Grimm J, Fan J, An L, Xue J, et al. Dosimetric investigation of accelerated partial breast irradiation (APBI) using CyberKnife. Med Phys 2012; 39: 6621-8. doi: 10.1118/1.4757616

26. Goggin LM, Descovich M, McGuinness C, Shiao S, Pouliot J, Park C. Dosimetric bomparison between 3-dimensional conformal and robotic SBRT treatment plans for accelerated partial breast radiotherapy. Technol Cancer Res Treatm 2016; 15: 437-45. doi: 10.1177/1533034615601280
27. Rault E, Lacornerie T, Dang H, Crop F, Lartigau E, Reynaert N, et al. Accelerated partial breast irradiation using robotic radiotherapy: a dosimetric comparison with tomotherapy and three-dimensional conformal radiotherapy. Radiat Oncol 2016; 11: 29. doi: 10.1186/s13014-016-0607-9

28. Bonfantini F, De Martin E, Giandini T, Fumagalli ML, Cavallo A, Pinzi V, et al. A dosimetric comparison between three different external photon beam techniques for accelerated partial breast irradiation. Clin Oncol 2018; 3: 1501.

29. Lee CY, Kim WC, Kim HJ, Lee J, Park S, Huh HD. Dosimetric plan comparison of accelerated partial breast irradiation (APBI) Using CyberKnife. Prog Med Phys 2018; 29: 73-80. doi: 10.14316/pmp.2018.29.2.73

30. Shahbazian H, Bakhshali R, Shamsi A, Bagheri A. Dosimetric analysis of breast cancer tumor bed boost: an interstitial brachytherapy vs. external beam radiation therapy comparison for deeply seated tumors. Brachytherapy 2020; 19: 264-74. doi: 10.1016/j.brachy.2019.10.008

31. Fan J, Hayes S, Freedman G, Anderson P, Li J, Wang L, et al. Planning the breast boost: dosimetric comparison of CyberKnife, photon mini tangents, IMRT, and electron techniques. Int J Radiat Oncol Biol Phys 2010; 78(3 Suppl): S788-9. doi: 10.1016/j.ijrobp.2010.07.1826

32. Stelczer G, Major T, Mészáros N, Polgár Cs, Pesznyák Cs. External beam accelerated partial breast irradiation: dosimetric assessment of conformal and three different intensity modulated techniques. Radiol Oncol 2019; 53: 123-30. doi:10.2478/raon-2019-0001

33. Anbumani S, Palled SR, Prabhakara GS, Nambiraj NA, Pichandia A Accelerated partial breast irradiation using external beam radiotherapy - a feasibility study based on dosimetric analysis. Rep Pract Onc Radiother 2012; 7: 200-6. doi: 10.1016/j.rpor.2012.04.002

34. Major T, Stelczer G, Pesznyák Cs, Mészáros N, Polgár Cs. Multicatheter interstitial brachytherapy versus intensity modulated external beam therapy for accelerated partial breast irradiation: a comparative treatment planning study with respect to dosimetry of organs at risk. Radiother Oncol 2017, 122: 17-23. doi: 10.1016/j.radonc.2016.08.003

35. Charaghvandi RK, den Hartogh MD, van Ommen ALN, de Vries WJH, Scholten $\mathrm{V}$, Moerland MA, et al. MRI-guided single fraction ablative radiotherapy for early-stage breast cancer: a brachytherapy versus volumetric modulated arc therapy dosimetry study. Radiother Oncol 2015; 117: 47782. doi: 10.1016/j.radonc.2015.09.023

36. Fröhlich G, Mészáros N, Smanykó V, Stelczer G, Herein A, Polgár Cs, et al. Is stereotactic CyberKnife radiotherapy or multicatheter HDR brachytherapy the better option dosimetrically for accelerated partial breast irradiation? Brachytherapy Nov 19, 2020; [Ahead of print]. doi: 10.1016/j. brachy.2020.10.003

37. Major T, Polgár Cs, Lövey K, Fröhlich G. Dosimetric characteristics of accelerated partial breast irradiation with CT image-based multicatheter interstitial brachytherapy: a single institution's experience. Brachytherapy 2011; 10: 421-6. doi: 10.1016/j.brachy.2010.12.006

38. Nath R, Anderson LL, Luxton G, Weaver KA, Williamson JF, Meigooni AS. Dosimetry of interstitial brachytherapy sources: Recommendations of the AAPM Radiation Therapy Committee Task Group No. 43. Med Phys 1995; 22: 209-34.

39. Guinot JL, Gonzalez-Perez V, Meszaros N, Major T, Najjari-Jamal D, GutierrezMiguelez C, et al; GEC-ESTRO Breast Working Group2. Very accelerated partial breast irradiation Phase I-II multicenter trial (VAPBI): feasibility and early results. Brachytherapy 2020; Nov 19, 2020; [Ahead of print]. doi: 10.1016/j. brachy.2020.10.010

40. Mészáros $\mathrm{N}$, Smanykó $\mathrm{V}$, Major $\mathrm{T}$, Stelczer $\mathrm{G}$, Jánváry L, Kovács $\mathrm{E}$, et al. Implementation of stereotactic accelerated partial breast irradiation using cyber-knife - technical considerations and early experiences of a phase II clinical study. Pathol Oncol Res 2020; 26: 2307-13. doi: 10.1007/s12253020-00821-3

41. Zhen X, Zhao B, Wang Z, Timmerman R, Spangler A, Kim N, et al. Comprehensive target geometric errors and margin assessment in stereotactic partial breast irradiation. Radiat Oncol 2017; 12: 151. doi: 10.1186/ s13014-017-0889-6

42. Baltas D, Kolotas C, Geramani K, Mould RF, loannidis G, Kekchidi M, et al. A conformal index (COIN) to evaluate implant quality and dose specification in brachytherapy. Int J Radiat Oncol Biol Phys 1998; 40: 515-24. doi: 10.1016/ s0360-3016(97)00732-3 
43. Rusthoven KE, Carter DL, Howell K, Kercher JM, Henkenberns P, Hunter KL, et al. Accelerated partial-breast intensitymodulated radiotherapy results in improved dose distribution when compared with three-dimensiona treatment-planning techniques. Int J Radiat Oncol Biol Phys 2008; 70: 296302. doi: 10.1016/j.jijrobp.2007.08.047

44. Moon SH, Shin KH, Kim TH, Yoon M, Park S, Lee DH, et al. Dosimetric comparison of four different external beam partial breast irradiation techniques: three-dimensional conformal radiotherapy, intensity-modulated radiotherapy, helical tomotherapy, and proton beam therapy. Radiother Oncol 2009; 9: 66-73. doi: 10.1016/j.radonc.2008.09.027

45. Qiu JJ, Chang Z, Horton JK, Wu QRJ, Yoo S, Yin FF. Dosimetric comparison of 3D conformal, IMRT, and V-MAT techniques for accelerated partialbreast irradiation (APBI). Med Dosim 2014; 39: 152-8. doi: 10.1016/j.meddos.2013.12.001

46. Major T, Niehoff P. Kovács Gy, Fodor J, Polgár Cs. Dosimetric comparisons between high dose rate interstitial and MammoSite ${ }^{\mathrm{TM}}$ balloon brachytherapy for breast cancer. Radiother Oncol 2006; 79: 321-8. doi: 10.1016/j. radonc.2006.05.005

47. Rahimi A, Thomas K, Spangler A, Rao R, Leitch M, Wooldridge R, et al. Preliminary results of a phase 1 dose-escalation trial for early-stage breast cancer using 5 -fraction stereotactic body radiation therapy for partial-breast irradiation. Int J Radiat Oncol Biol Phys 2017; 98: 196-205.e2. doi: 10.1016/j ijrobp.2017.01.020

48. Bondiau PY, Bahadoran P, Lallement M, Birtwisle-Peyrottes I, Chapellier C, Chamorey E, et al. Robotic stereotactic radioablation concomitant with neoadjuvant chemotherapy for breast tumors. Int J Radiat Oncol Biol Phys 2009; 75: 1041-7. doi: 10.1016/j.jijrobp.2008.12.037

49. Vermeulen S, Cotrutz C, Morris A, Meier R, Buchanan C, Dawson P, et al. Accelerated partial breast irradiation: using the CyberKnife as the radiation delivery platform in the treatment of early breast cancer. Front Oncol 2011; 1: 43. doi: $10.3389 /$ fonc. 2011.00043

50. NSABP PROTOCOL B-39, RTOG PROTOCOL 0413. [internet]. [cited 2020 May 27]. Available at: http://rpc.mdanderson.org/rpc/credentialing/files/ B39_Protocol1.pdf

51. Vermeulen SS, Haas JA. CyberKnife stereotactic body radiotherapy and CyberKnife accelerated partial breast irradiation for the treatment of early breast cancer. Transl Cancer Res 2014; 3: 295-302. doi: 10.3978/j.issn.2218 676X.2014.07.06

52. Obayomi-Davies O, Kole TP, Oppong B, Rudra S, Makariou EV, Campbell LD, et al. Stereotactic accelerated partial breast irradiation for early-stage breast cancer: rationale, feasibility, and early experience using the cyberknife radiosurgery delivery platform. Front Oncol 2016; 6: 129. doi: 10.3389/ fonc.2016.00129

53. Lozza L, Fariselli L, Sandri M, Rampa M, Pinzi V, Carmen De Santis M, et al. Partial breast irradiation with CyberKnife after breast conserving surgery: a pilot study in early breast cancer. Radiat Oncol 2018; 13: 49. doi: 10.1186/ s13014-018-0991-4

54. Lee WH, Chang JS, Kim MJ, Park VY, Yoon JH, Kim SY, et al. First experience in Korea of stereotactic partial breast irradiation for low-risk early-stage breast cancer. Front Oncol 2020; 10: 672. doi: 10.3389/fonc.2020.00672

55. Fröhlich G, Geszti Gy, Vízkeleti J, Ágoston P, Polgár Cs, Major T. Dosimetric comparison of inverse optimisation methods versus forward optimisation in HDR brachytherapy of breast, cervical and prostate cancer. Strahlenther Onkol 2019; 195: 991-1000. doi: 10.1007/s00066-019-01513-x

56. Major T, Fröhlich G, Mészáros N, Smanykó V, Polgár CS. Does inverse planning improve the plan quality in interstitial high dose rate breast brachytherapy? J Contemp Brachytherapy 2020; 12: 166-74. doi: 10.5114/ jcb.2020.94584

57. Sanjay R, Jaiteerth SA, Moonseong MS, Harish KM, Wainwright J, Michae RK, et al. Quantifying IOHDR brachytherapy underdosage resulting from an incomplete scatter environment. Radiat Oncol Biol Phys 2005; 61: 1582-6. doi: 10.1016/j.ijrobp.2004.10.002 